\title{
A SIR Mathematical Model of Dengue Transmission and Its Simulation
}

\author{
Asmaidi $^{1}$, Paian Sianturi ${ }^{2}$, Endar Hasafah Nugrahani ${ }^{3}$ \\ I'Department of Mathematics Faculty of Mathematics and Natural Science, Bogor Agricultural University, \\ Indonesia) \\ ${ }_{2}^{2}$ (Department of Mathematics Faculty of Mathematics and Natural Science, Bogor Agricultural University, \\ Indonesia) \\ ${ }^{3}$ (Department of Mathematics Faculty of Mathematics and Natural Science, Bogor Agricultural University, \\ Indonesia)
}

\begin{abstract}
In this paper developed a mathematical model of the spread of dengue hemorrhagic fever (DHF) SIR type, where SIR is an abbreviation of susceptible (S), infected (I) and recovered (R). Results of analysis and simulation obtained two fixed points, namely the disease-free quilibrium and endemic equilibrium. Human population, mosquitoes and mosquito eggs stable around the disease-free quilibrium when $\mathcal{R}_{0}<1$ and stable around the endemic equilibrium point when $\mathcal{R}_{0}>1$. Increased of mosquitoes mortality rate can reduce the value of the basic reproduction number.
\end{abstract}

Keywords - mathematical models, basic reproductive number, disease-free quilibrium, endemic equilibrium, numerical simulations

\section{Introduction}

Mathematical model of dengue transmission considers the temperature factor ever conducted by Massad et al. (2011) and Amaku et al. (2013), by developing the SIR model of humans-mosquitoes-mosquito eggs. SIR is an abbreviation of susceptible (S), Infected (I) and recovered (R). In the SIR model of mosquitoeshumans-mosquito eggs, the human populations are made up of three populations, namely susceptible humans, infected humans and recovered humans. Mosquito population consists of three populations, namely susceptible mosquitoes, latent mosquitoes and infected mosquitoes. The population of the mosquito eggs consists of two populations, namely healthy mosquito eggs and infected mosquito eggs.

In the model formulated by [5], it was assumed the transfer rate of infected mosquitoes become a latent mosquitoes involves time delay $(\tau)$ as the incubation periodic the mosquito. Moreover, [5] assumes a healthy mosquito eggs produced by infected mosquitoes and susceptible mosquitoes, while the infected mosquito eggs only produced by infected mosquitoes. Temperature factors were used [5] in producing susceptible mosquitoes and infected mosquitoes from eggs is not constant.

In the model [1], assumed to be a latent transfer rate of mosquitoes infected mosquito does not involve a time delay and a healthy mosquito eggs are produced by third compartment mosquitoes, while the mosquito is infected eggs produced by infected mosquitoes and mosquitoes latent. Temperature factors were used [1] is a constant.

Based on these two studies, the model of SIR humans-mosquitoes-mosquito eggs modified assuming the transfer rate latent mosquitoes become infected mosquito does not involve a time delay, as the assumptions used [1]. Healthy mosquito eggs and infected mosquito eggs production rate used [5] assumptions. In addition, the temperature factor used in producing susceptible mosquitoes and infected mosquitoes from eggs. Temperature factors are the same as those used in the study [1].

\section{Mathematical Model}

In $S I R$ model human-mosquito-eggs, human population is divided into susceptible humans $\left(S_{H}\right)$, infected humans $\left(I_{H}\right)$, dan recovered humans $\left(R_{H}\right)$, with total human population $N_{H}=S_{H}+I_{H}+R_{H}$. Mosquito is divided into susceptible mosquitoes $\left(S_{M}\right)$, latent mosquitoes $\left(L_{M}\right)$, and infected mosquitoes $\left(I_{M}\right)$, with total mosquito $N_{M}=S_{M}+L_{M}+I_{M}$. Egg population is divided into non-infected eggs $\left(S_{E}\right)$ and infected eggs $\left(I_{E}\right)$, with total egg population $N_{E}=S_{E}+I_{E}$. 


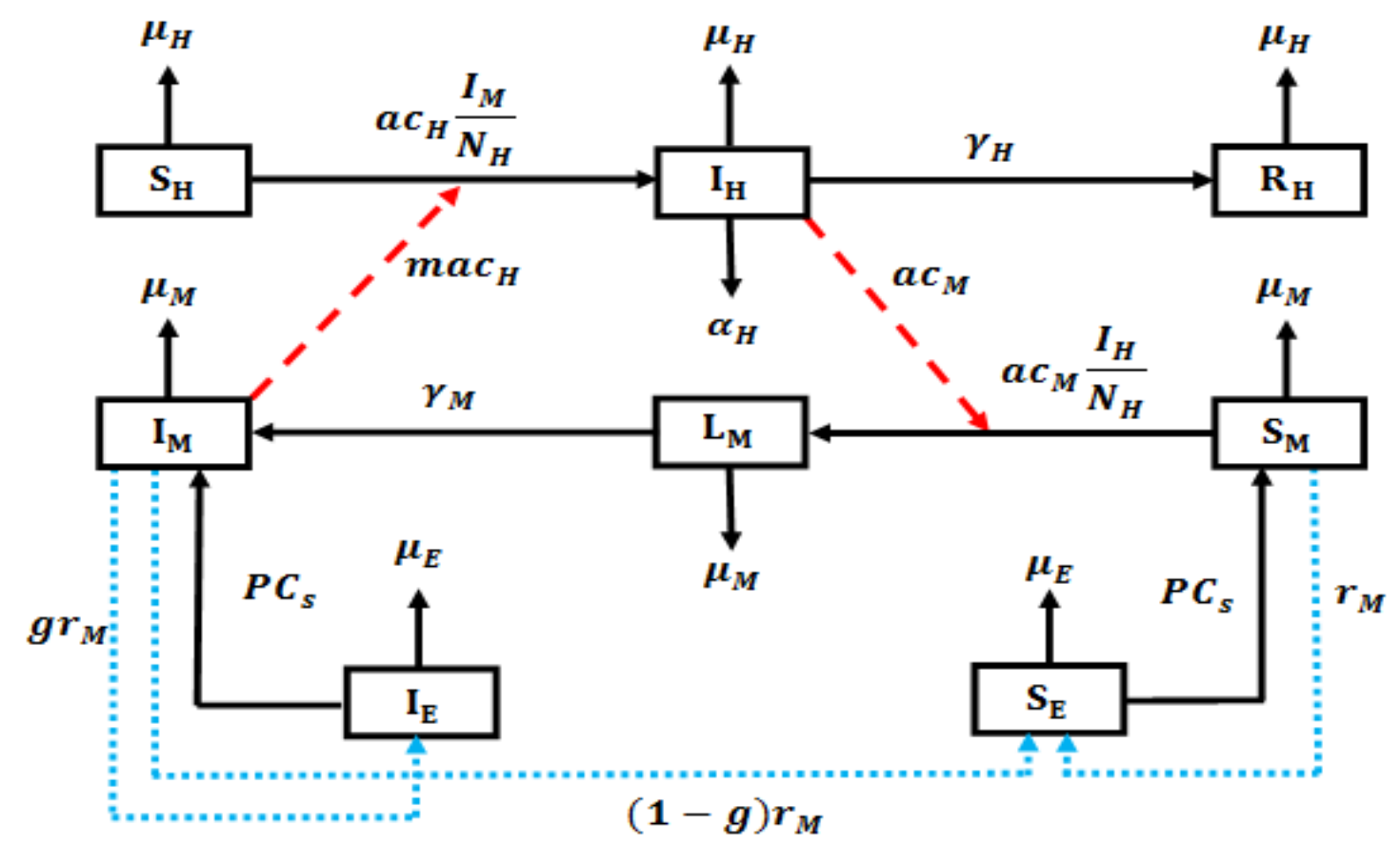

Figure 1. Model modification

Basedon compartmenton Figure 1 the system of differential equations is obtained for each compartment, as follows:

$$
\begin{aligned}
\frac{d S_{H}}{d t} & =r_{H} N_{H}\left(1-\frac{N_{H}}{k_{H}}\right)-\left(a c_{H} \frac{I_{M}}{N_{H}}+\mu_{H}\right) S_{H} \\
\frac{d I_{H}}{d t} & =a c_{H} \frac{I_{M}}{N_{H}} S_{H}-\left(\alpha_{H}+\mu_{H}+\gamma_{H}\right) I_{H} \\
\frac{d R_{H}}{d t} & =\gamma_{H} I_{H}-\mu_{H} R_{H} \\
\frac{d S_{M}}{d t} & =P C_{S} S_{E}-a c_{M} \frac{I_{H}}{N_{H}} S_{M}-\mu_{M} S_{M} \\
\frac{d L_{M}}{d t} & =a c_{M} \frac{I_{H}}{N_{H}} S_{M}-\gamma_{M} L_{M}-\mu_{M} L_{M} \\
\frac{d I_{M}}{d t} & =\gamma_{M} L_{M}-\mu_{M} I_{M}+P C_{S} I_{E} \\
\frac{d S_{E}}{d t} & =\left(r_{M} S_{M}+(1-g) r_{M} I_{M}\right)\left(1-\frac{\left(S_{E}+I_{E}\right)}{k_{E}}\right)-\mu_{E} S_{E}-P C_{S} S_{E} \\
\frac{d I_{E}}{d t} & =g r_{M} I_{M}\left(1-\frac{\left(S_{E}+I_{E}\right)}{k_{E}}\right)-\left(\mu_{E}+P C_{s}\right) I_{E} .
\end{aligned}
$$

The infection rate from infected mosquitoes to susceptible humansis $\beta_{H}=m a b_{H}$, where $m$ is the ratio of total mosquito population to the total population of humans, so that the transfer rate of susceptible humans become infected human as follows

$\frac{N_{M} a b_{H} \frac{I_{M}}{N_{M}}}{N_{H}}=a b_{H} \frac{I_{M}}{N_{H}}=m a b_{H} \frac{I_{M}}{N_{M}}=\beta_{H} \frac{I_{M}}{N_{M}}$.

The infection from infected humans to susceptible mosquitoes is $\beta_{M}=a b_{M}$, so that the transfer rate of susceptible mosquitoes become latent mosquitoes as follows 
$\frac{N_{M} a b_{M} \frac{I_{H}}{N_{H}}}{N_{M}}=a b_{M} \frac{I_{H}}{N_{H}}=\beta_{M} \frac{I_{H}}{N_{H}}$.

The temperature factor used in mosquito egg hatching, ie

$C_{S}=0.07$

Several parameters used in modificated model are, fraction of infective bites from an infected human $\left(c_{H}\right)$, fraction of infective bites from an infected mosquitoes $\left(c_{M}\right)$, dengue induced mortality in humans $\left(\alpha_{H}\right)$, humans recovery rate $\left(\gamma_{H}\right)$, latency rate in mosquitoes $\left(\gamma_{M}\right)$, humans natural mortality rate $\left(\mu_{H}\right)$, natural mortality rate of eggs $\left(\mu_{E}\right)$, birth rate of humans $\left(r_{H}\right)$, humans carrying capacity $\left(k_{H}\right)$, infected eggs hatching rate $(P)$, infected eggs hatching rate $(g)$, oviposition rate $\left(r_{M}\right)$, eggs carrying capacity $\left(k_{E}\right)$, climatic factor $\left(C_{s}\right),[1]$ and [5].

To facilitate the analysis of the equation (1), simplified by making a comparison of each population to the total population, ie

$$
S_{h}=\frac{S_{H}}{N_{H}} ; I_{h}=\frac{I_{H}}{N_{H}} ; R_{h}=\frac{R_{H}}{N_{H}} ; S_{m}=\frac{S_{M}}{N_{M}} ; L_{m}=\frac{L_{M}}{N_{M}} ; I_{m}=\frac{I_{M}}{N_{M}} ; S_{e}=\frac{S_{E}}{N_{E}} ; I_{e}=\frac{I_{E}}{N_{E}},
$$

Then the system (5) is substituted into the system (1) to obtain a new differential equation system of five dimensions, namely

$$
\begin{aligned}
& \frac{d S_{h}}{d t}=r_{H}\left(1-\frac{N_{H}}{k_{H}}\right)-\left(m a c_{H} I_{m}+\mu_{H}\right) S_{h} \\
& \frac{d I_{h}}{d t}=m a c_{H} I_{m} S_{h}-\left(\alpha_{H}+\mu_{H}+\gamma_{H}\right) I_{h} \\
& \frac{d L_{m}}{d t}=a c_{M} I_{h}\left(1-L_{m}-I_{m}\right)-\left(\mu_{M}+\gamma_{M}\right) L_{m} \\
& \frac{d I_{m}}{d t}=\gamma_{M} L_{m}-\mu_{M} I_{m}+P C_{s} w I_{e} \\
& \frac{d I_{e}}{d t}=\frac{g r_{M} I_{m}}{w}\left(1-\frac{N_{E}}{k_{E}}\right)-\left(\mu_{E}+P C_{s}\right) I_{e}
\end{aligned}
$$

with

$$
S_{h}+I_{h}+R_{h}=1, S_{m}+L_{m}+I_{m}=1 \text { and } S_{e}+I_{e}=1,
$$

where $w$ is the ratio of total mosquito eggs population to the total mosquitoes population. By taking the fifth equation (6), then the fixed point, the basic reproduction number, the stability of the fixed point and the population dynamics of the modified model can be determined.

\section{III. result and discussion}

\subsection{Equilibrium State}

The equation used to determine the fixed point is equation (6). Determine the fixed point system of differential equations of the model modifications done in the following way:

\subsubsection{Disease-Free Equilibrium}

$$
\frac{d S_{h}}{d t}=0, \frac{d I_{h}}{d t}=0, \frac{d L_{m}}{d t}=0, \frac{d I_{m}}{d t}=0 \text { and } \frac{d I_{e}}{d t}=0 .
$$

$$
\boldsymbol{T}_{\mathbf{0}}\left(S_{h}, I_{h}, L_{m}, I_{m}, I_{e}\right)=\left(\frac{r_{h}\left(1-\frac{N_{H}}{k_{H}}\right)}{\mu_{h}}, 0,0,0,0\right) \text {. }
$$

\subsubsection{Endemic Equilibrium}

$\boldsymbol{T}_{\mathbf{1}}\left(S_{h}^{*}, I_{h}^{*}, L_{m}^{*}, I_{m}^{*}, I_{e}^{*}\right)$, yaitu 


$$
\begin{aligned}
& S_{h}^{*}=\frac{\left(k_{H}-N_{H}\right) r_{H}}{k_{H}\left(a m c_{H} I_{m}^{*}+\mu_{H}\right)} ; \quad I_{h}^{*}=\frac{a m c_{H} I_{m}^{*} S_{h}^{*}}{\left(\alpha_{H}+\mu_{H}+\gamma_{H}\right)} ; \quad L_{m}^{*}=\frac{a c_{M} I_{h}^{*}\left(I_{m}^{*}-1\right)}{a c_{M} I_{h}^{*}+\gamma_{M}+\mu_{M}} \\
& I_{m}^{*}=\frac{P C_{s} w I_{e}^{*}+\gamma_{M} L_{m}^{*}}{\mu_{M}} ; I_{e}^{*}=\frac{g r_{M} I_{m}^{*}\left(\left(k_{E}-N_{E}\right)\right.}{k_{E} w\left(P C_{s}+\mu_{E}\right)}
\end{aligned}
$$

\subsection{Basic Reproduction Numbers}

Basic reproduction number is the expectation of number susceptible become infected during the course of infection. According to [3], the basic reproduction number can be determined by using the equation that contains the infection. The system of differential equations that are used to determine the basic reproduction number, ie equation (2), (3), (4), and (5) contained in equation (6). The approach used to determine the basic reproduction number as described by [3], using the next generation matrix $\boldsymbol{G}$ is defined as follows:

$$
\boldsymbol{G}=\boldsymbol{F} \boldsymbol{V}^{-\mathbf{1}},
$$

Based on the analysis obtained four eigenvalues, in which the dominant eigen values of the matrix $\boldsymbol{G}$, ie

$$
\mathcal{R}_{0}=\frac{a \sqrt{c_{H}} \sqrt{c_{M}} \sqrt{m} \sqrt{k_{H}-N_{H}} \sqrt{r_{H}} \sqrt{\gamma_{M}}}{\sqrt{k_{H} \mu_{H}\left(\alpha_{H}+\mu_{H}+\gamma_{H}\right) \mu_{M}\left(\mu_{M}+\gamma_{M}\right)}}
$$

\subsection{Population Dynamics Simulation of Dengue Transmission}

In the subsequent discussion made observations on the nature of the stability of each fixed point through numerical simulation. This simulation is done to see that the system will be stable around the free disease fixed point when $\mathcal{R}_{0}<1$ and stable around the endemic fixed point when $\mathcal{R}_{0}>1$. In addition, the simulation was also conducted to see the effect of the death rate of mosquitoes to the disease spread in the population. The parameter values used in the simulation are shown on Table 2.

Table 2 Parameters Value

\begin{tabular}{cc}
\hline Parameters & Value \\
\hline$c_{H}$ & 0.6 \\
$c_{M}$ & 0.6 \\
$\alpha_{H}$ & 0.001 \\
$\gamma_{H}$ & 0.143 \\
$\gamma_{M}$ & 0.143 \\
$\mu_{H}$ & $3.5 \times 10^{-5}$ \\
$\mu_{E}$ & 0.5 \\
$r_{H}$ & $2.4 \times 10^{-5}$ \\
$k_{H}$ & $4 \times 10^{5}$ \\
$P$ & 0.15 \\
$g$ & 0.1 \\
$r_{M}$ & 50 \\
$k_{E}$ & $10^{6}$ \\
$C_{s}$ & 0.07 \\
\hline
\end{tabular}

Source: [1], [2], [4], and [5]

\subsubsection{Population Dinamics for $\mathcal{R}_{0}<1$}

The values of the parameters used in the simulation for condition $\mathcal{R}_{0}<1$ can be seen in Table 2, with the total population of each $N_{H}=10, N_{M}=20$ and $N_{E}=70$. Further, we discuss the behavior of the population for conditions $\mathcal{R}_{0}<1$.

System (6) has a disease-free equilibrium for conditions $\mathcal{R}_{0}<1$ which can be shown by simulation. The fixed point is obtained using the parameter values listed in Table 2 with the value of $\mathcal{R}_{0}=0.31$.. The disease-free equilibrium, namely

$$
\boldsymbol{T}_{\mathbf{0}}\left(S_{h}, I_{h}, L_{m}, I_{m}, I_{e}\right)=(0.685697,0,0,0,0) .
$$


The parameter values used to simulate the average level of mosquito bites $(a)$ is 0146 , [1]. The initial values used in the simulation is $S_{h}=0.85, I_{h}=0.15, L_{m}=0.2, I_{m}=0.1$ and $I_{e}=0$. The behavior of the population for conditions $\mathcal{R}_{0}<1$ is shown in Figure 2,

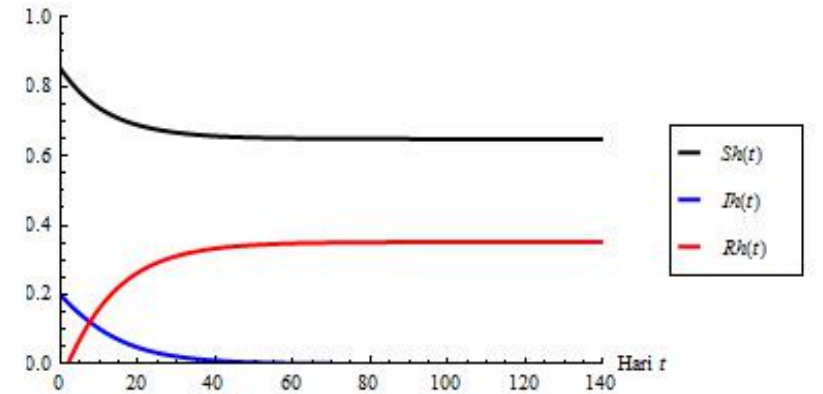

(a)

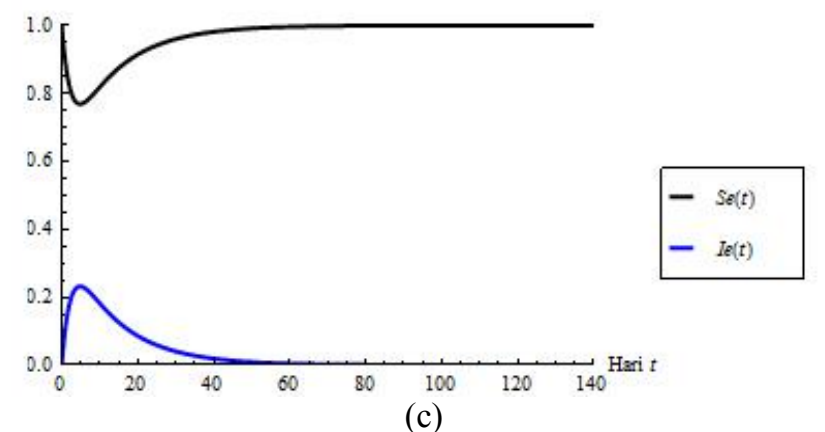

Figure 2 Humans (a), Mosquitoes (b) and Mosquito eggs (c) population for $\mathcal{R}_{0}<1$

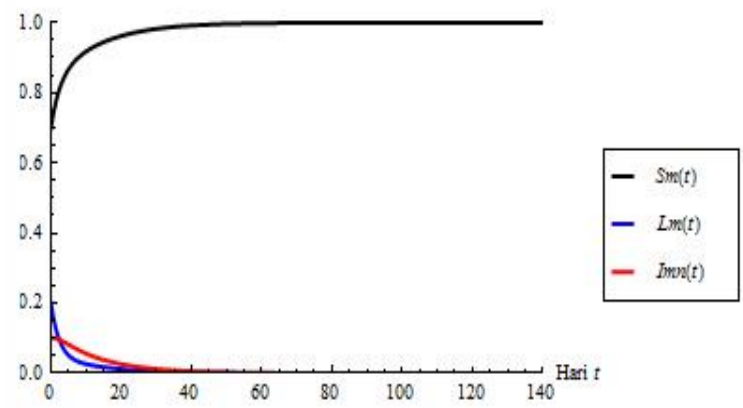

(b)

Figure 2 illustrates the humans, mosquitoes and mosquito eggs population. Figure (a) describes the behavior of humans populations. Susceptible human populations $\left(S_{h}\right)$ decreased from an initial value, then stabilized at around $S_{h}=0.685697$. The infected human population $\left(I_{h}\right)$ decreased from an initial value, then stabilized at around $I_{h}=0$, while the recovered human population $\left(R_{h}\right)$ increased from an initial value, then stabilized at around $R_{h}=1-S_{h}-I_{h}=0.314303$.

Figure (b) describes the mosquito population, where the latent mosquitoes population $\left(L_{m}\right)$ decreased from an initial value, then stabilized at around $L_{m}=0$, the population of infected mosquitoes $\left(I_{m}\right)$ decreased from an initial value, then stabilized at around $I_{m}=0$, while the susceptible mosquitoes population $\left(S_{m}\right)$ increased from an initial value, then stabilized at around $S_{m}=1-L_{m}-I_{m}=1$.

Picture (c) explain the mosquito eggs population, where the infected mosquito eggs population $\left(I_{e}\right)$ increased from an initial value, then decreased and finally stabilized at around $I_{e}=0$, while the population of healthy mosquito eggs $\left(S_{e}\right)$ decreased from an initial value, then increases and finally stable at around $S_{e}=1-$ $I_{e}=1$. Based on the simulation it can be concluded that each population towards a free disease fixed point or in other words the human population, mosquitoes and mosquito eggs stable around the fixed point $(0.685697,0,0,0,0)$.

\subsubsection{Population Dinamics for $\mathcal{R}_{0}>1$}

System (6) has a endemic fixed point when $\mathcal{R}_{0}>1$ which can be shown by simulation. The fixed point is obtained using the parameter values listed in Table 2 with the value of $\mathcal{R}_{0}=7.93$. Based on the parameter values in Table 2 obtained endemic fixed point, ie

$$
\boldsymbol{T}_{\mathbf{1}}\left(S_{h}^{*}, I_{h}^{*}, L_{m}^{*}, I_{m}^{*}, I_{e}^{*}\right)=(0.006656,0.000165,0.000901,0.000804,0.002250) .
$$

The average value of the level mosquito bites parameter $(a)$ used is 3.7, Massad et al. (2011) with parameters such as the mosquito mortality rate in the previous simulation $\mu_{M}=0.263$. The figure 3 shows the behavior of each population in the existing state of the disease. 


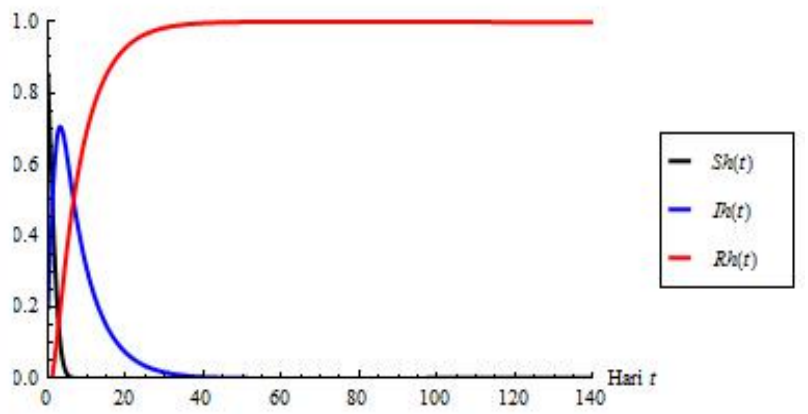

(a)

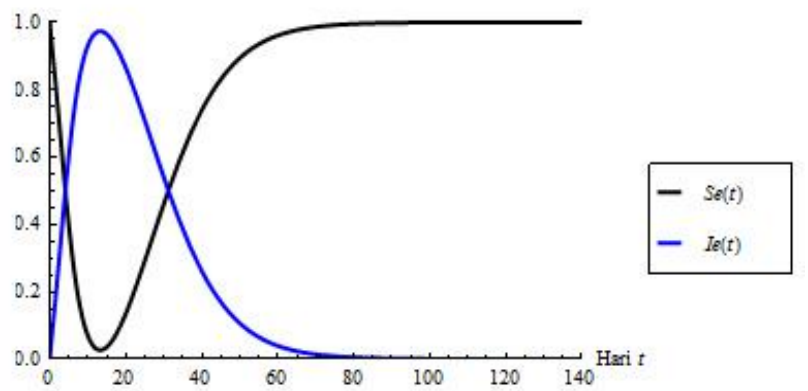

(c)

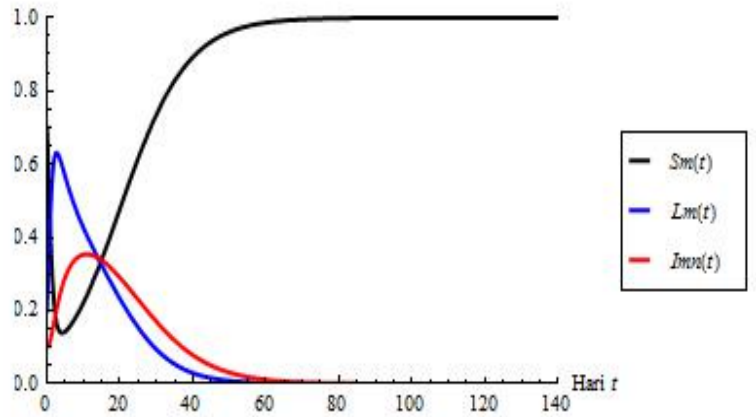

(b)

Figure 3 Humans (a), Mosquitoes (b) and Mosquito eggs (c) population for $\mathcal{R}_{0}>1$

Figure 3 above describes the human population, mosquitoes and mosquito eggs. The simulation results in Figure (a) can be noted that the susceptible human population $\left(S_{h}\right)$ decreased from an initial value, then stabilized at around $S_{h}=0.006656$, the infected human population $\left(I_{h}\right)$ increased from an initial value, then decreased and finally stabilized at around $I_{h}=0.000165$, while the recovered human population $\left(R_{h}\right)$ increased from an initial value, then stabilized at around $R_{h}=1-S_{h}-I_{h}=0.993179$.

Figure (b) describes the mosquito population, where the population of latent mosquitoes $\left(L_{m}\right)$ increased from an initial value, then decreased and finally stabilized at around $L_{m}=0.000901$, the infected mosquitoes population $\left(I_{m}\right)$ increased from an initial value, then decreased and finally stabilized at around $I_{m}=0.000804$, while the susceptible mosquitoes population $\left(S_{m}\right)$ decreased from an initial value, then increased and finally stabilized at around $S_{m}=1-L_{m}-I_{m}=0.998295$.

Picture (c) explain the mosquito eggs population, where the infected mosquito eggs population $\left(I_{e}\right)$ increased from an initial value, then decreased and finally stabilized at around $I_{e}=0.002250$, while the population of healthy mosquito eggs $\left(S_{e}\right)$ decreased from an initial value, then increases and finally stable at around $S_{e}=1-I_{e}=0.99775$. Based on the simulation it can be concluded that each population is stable around a endemic fixed point, namely $(0.006656,0.000165,0.000901,0.000804,0.002250)$.

The next stage is the simulation of the mosquito mortality rate influence $\left(\mu_{M}\right)$ against the spread of disease in populations. In this simulation, the parameters $\left(\mu_{M}\right)$ taken on the interval [0163, 0563] with step 0.1 which still meets the conditions $\mathcal{R}_{0}<1$.

\subsection{Mosquito Mortality Rate Simulation}

Simulation is necessary to see the effect of the mosquito mortality rate to the spread of the disease in the population. Moreover, it will be shown that an increase in mosquito mortality rate can decrease the value of the basic reproduction number $\left(\mathcal{R}_{0}\right)$, defined in equation (8). There are 4 values $\mu_{M}$ taken on the interval [0.263, 0.563 ] with step 0.1 . The values of other parameters in Table 5 , by taking the average value of the mosquito bites $a=0.146$, [1]. Table 3 below shows the current value of $\mathcal{R}_{0}$ on mosquito mortality rate increased. 
Table 3 Value $\mathcal{R}_{0}$ with $\mu_{M}$ parameters enhanced and other parameters remain

\begin{tabular}{|c|c|c|}
\hline \multicolumn{2}{|c|}{ Parameters Value } & \multirow{2}{*}{$\mathcal{R}_{0}$} \\
\hline$a$ & $\mu_{M}$ & \\
\hline 0.146 & 0.263 & 0.31 \\
\hline 0.146 & 0.363 & 0.23 \\
\hline 0.146 & 0.463 & 0.19 \\
\hline 0.146 & 0.563 & 0.16 \\
\hline
\end{tabular}

Based on Table 3, if the mortality rate of mosquitoes increased the basic reproduction number will decrease. The decline in basic reproduction number can help reduce the rate of spread of disease in the population. Furthermore, the simulation effect of the death rate of mosquitoes that aims to observe the changes in the human populations, mosquitoes and mosquito eggs populations, if the mosquito death rate increased. Figure 4 shows the effects that occur in the human population if the mosquito death rate increased.

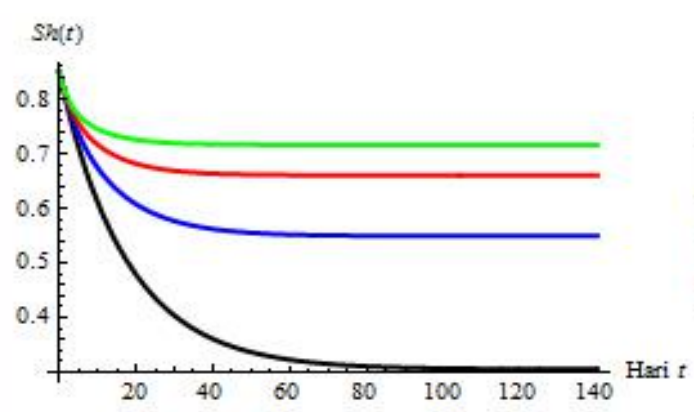

(a)

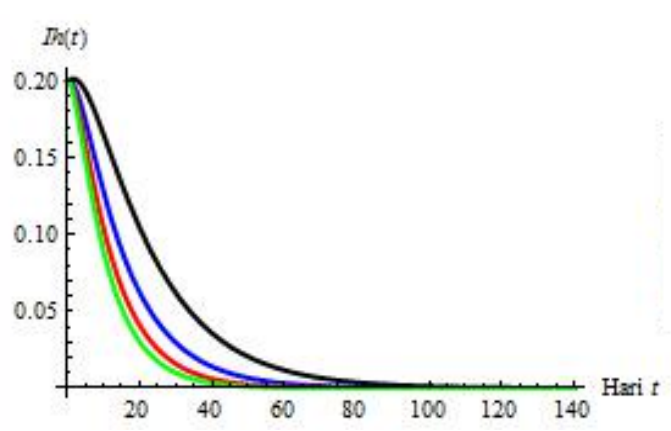

(b)

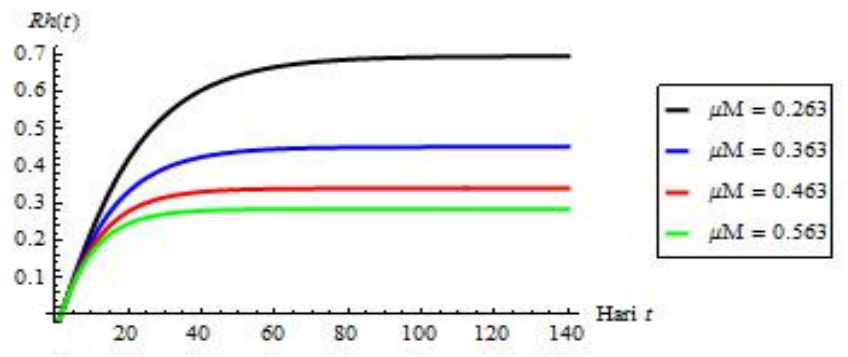

(c)

Figure 4 Human population

Figure $4 a-4 c$ describes the change of each human population, with a mortality rate of mosquitoe enhanced and other parameters fixed. Susceptible human population increases when the mosquito death rate increased. The infected human population is reduced at the beginning of the simulation when the mosquito death rate increased, and at the end of the simulation also reduced but not too different. Recovered human population reduced when the mosquito death rate increased.

When mosquitoe mortality rate increased it means reducing the infected mosquito population. Reduction in the infected mosquito population cause infection rates occur in susceptible human population is reduced, thus increasing the susceptible human population. The increasing of susceptible human population causes the transfer rate of susceptible humans become infected humans is reduced at the beginning of the simulation, and but at the end of the simulation also reduced but not too different. Reduction in the infected human population cause the transfer rate from infected human becomes recoverd humans also reduced. In addition, the increase or decrease in the infected human population gave effect to the mosquito population. The effect in the mosquito population can be seen in Figure 5. 


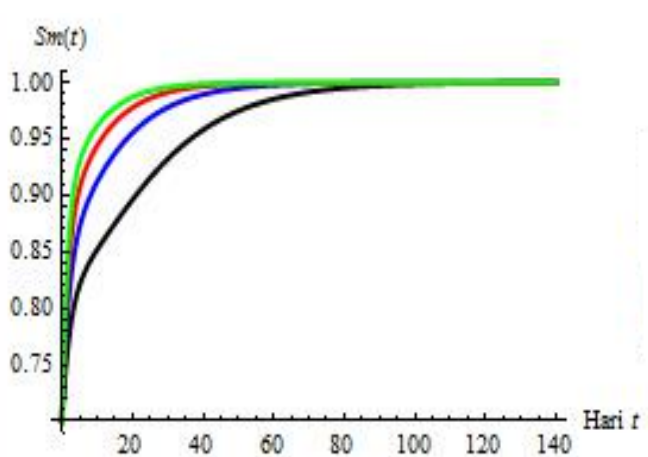

(a)

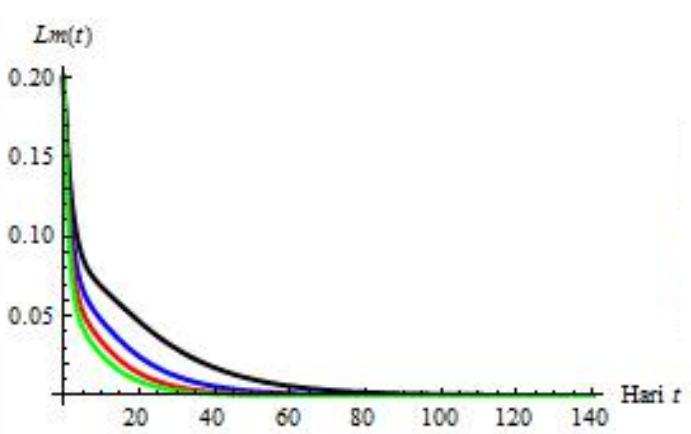

(b)

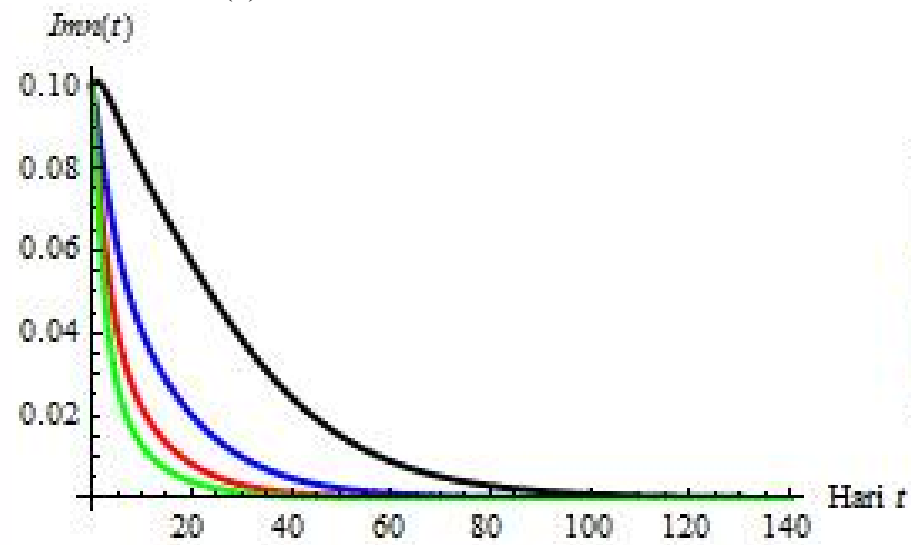

(c)

Figure 5 Mosquito population

Figure 5a-5c shows the changes of each mosquito population, with a mortality rate of mosquitoes enhanced and other parameters remain. Susceptible mosquito population at the beginning of the simulation increases when the mosquito death rate increased, and at the end of the simulation also increase but not too different. Latent mosquito population at the beginning of the simulation is reduced when the mosquito death rate increased, and at the end of the simulation also reduced but not too different. The same thing happens to the infected mosquito population, where the number of infected mosquito population at the beginning of the simulation is reduced when the mosquito death rate increased, and at the end of the simulation also reduced but not too different.

Susceptible mosquito populations increase at the beginning of the simulation due to the reduction in the infected human population, in which at the beginning of the simulation the infected human population reduced when the mosquito mortality rate increased, so that the rate of infection occurs in susceptible mosquito population is reduced. Therefore, the transfer rate of susceptible mosquito populations become latent mosquitoes at the beginning of the simulation is reduced, consequently the transfer rate of latent mosquitoes becom einfected mosquito is also reduced.

Susceptible mosquito population at the end of the simulation increased when mosquito mortality rate increase but not too different. This is due to the increase in infected human population at the end of the simulation when increased of mosquito mortality rate is not too different. When susceptible mosquito populations increase, causes the transfer of susceptible mosquito populations become latent mosquitoes reduced, reduced of latent mosquito population is no ttoo different. As a result, the transfer rate of latent mosquitoes become infected mosquitoes at the end of the simulationis also reduced by the amount that is not too different.

Increase or decrease of the population gave effect to the population of mosquito eggs. The effect that occurs in a population of mosquito eggs can be observed in Figure 6. 


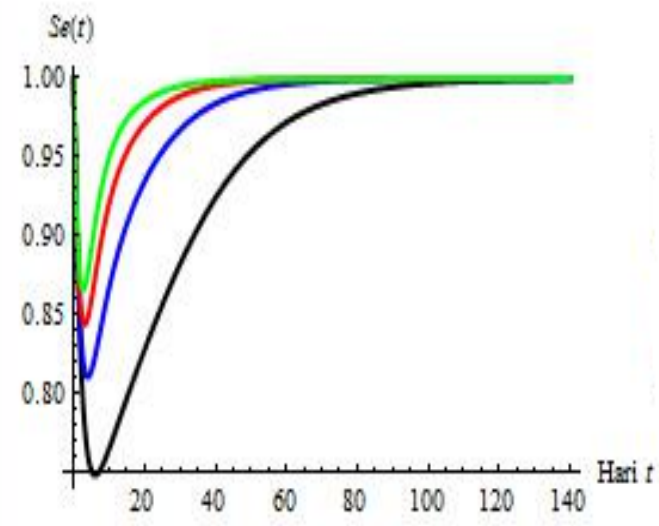

(a)

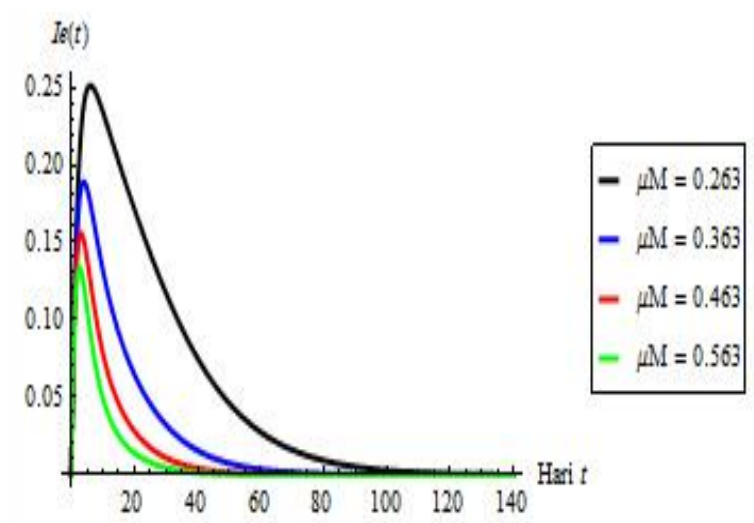

(b)

Figure 6 Eggs mosquito population

Figure $6 \mathrm{a}-6 \mathrm{~b}$ shows the population changes each mosqueto eggs when mosqueto mortality rate increased and other parameters remain. Healthy mosqueto eggs populations produced by infected mosquetoes and susceptible mosquitoes, but the healthy mosquito eggs population produced more by susceptible mosquitoes. Therefore, the increase in the healthy mosquito eggs population at the beginning of the simulation when mosquito mortality rate increased, caused by increasing of susceptible mosque to populations. Healthy mosquito eggs population at the end of the simulation increases with the number that is not too different. An other thing occurs in the infected mosquito eggs population, where the infected mosquito eggs population is only produced by infected mosquitoes. Therefore, the reduction in the population of infected mosquito eggs at the beginning of the simulation when mosquito mortality rate increased caused by the reduced number of infected mosquito populations. At the end of the simulation infected mosquito eggs population reduced by the amount that is not too different. This is caused by the infected mosquito population at the end of the simulation is reduced by the amount that is not too different.

\section{Conclusion}

Based on the analysis and discussion are carried out on the modified model can be concluded that,

1. There are two fixed point obtained, namely the disease-free equilibrium $\boldsymbol{T}_{\mathbf{0}}\left(S_{h}, I_{h}, L_{m}, I_{m}, I_{e}\right)$ and the endemic equilibrium $\boldsymbol{T}_{\mathbf{1}}\left(S_{h}^{*}, I_{h}^{*}, L_{m}^{*}, I_{m}^{*}, I_{e}^{*}\right)$. At the fixed point there are five populations that is susceptible humans $\left(S_{h}\right)$, infected humans $\left(I_{h}\right)$, latent mosquitoes $\left(L_{m}\right)$, infected mosquitoes $\left(I_{m}\right)$ and infected mosque toes eggs $\left(I_{e}\right)$.

2. Disease-free equilibrium stable when $\mathcal{R}_{0}<1$, while the endemic equilibrium table when $\mathcal{R}_{0}>1$.

3. Simulation results show that with the increasing mortality rate of mosquitoes, the basic reproduction number will decrease, so it can help reduce the rate of spread of the disease in the population.

4. The simulation results by changing the mosquito mortality rate $\left(\mu_{M}\right)$ give effect to the human population, mosquitoes and mosquito eggs, which

a. Effect occurs in human populations when mosquito mortality rate increased are susceptible human population increases, the infected human population at the beginning of the simulation decreases when mosquito mortality rate increased, but at the end of the simulation is also reduced by the amount that is not too different and recovered humans population is reduced.

b. Effect occurs in the mosquito population are susceptible mosquitoes population at the beginning of the simulation increases when mosquito mortality rate increased, and at the end of the simulation also increase but not too different. Latent mosquito population and infected mosquitoes reduced when mosqueto mortality rate in creased, but at the end of the simulation is also reduced by the amount that is not too different.

c. Effec toccurs in the mosque to egg spopulations are the healthy mosque to eggs population at the beginning of the simulation increases when mosque to mortality rate increased, and at the end of the simulation also in crease but not too different, while the population of infected mosquetoes eggs at the beginning of the simulation decreases when mosquito mortality rate increased, and at the end of the simulation is also reduced by the amount that is not too different. 


\section{References}

[1] Amaku M, Coutinho FAB, Raimundo SM, Lopez LF, Burattini MN, Massad E, $A$ Comparative Analysis of the Relative Efficacy of Vektor-Control Strategies Against Dengue Fever, Biomedical, DOI 10.1007/s11538-014-9939-5, 2013.

[2] Burattini MN, Chen M, Chow A, Coutinho FAB, Goh KT, Lopez LF, MA S, Massad E, Modelling the control strategies against dengue in Singapore, Epidemiol Infect, 2007, doi: 10.1017/S0950268807008667.

[3] Jones JH, 2007. Note on $\mathcal{R}_{0}, 2007$, Stanford.Department of Anthropological Sciences Stanford University.

[4] Jumadi, Model matematika penyebaran penyakit demam berdarah dengue, 2007, Bogor: Institut Pertanian Bogor.

[5] Massad E, Coutinho FA, Lopez LF, da Silva DR, Modeling the Impact of Global Warming on Vektor-Borne Infections, ScienceDirect, 8(2), 2011, 169-199 